\title{
A prospective study on corneal complications of small incision cataract surgery conducted in rural hospital
}

\author{
KriapliniS.H. ${ }^{1}$, Kumar $\mathbf{A}^{2}$ \\ ${ }^{1}$ Dr. Kripalini Soonthodu Hoovayya, Assistant Professor, Department of Ophthalmology, K.V.G Medical College and \\ Hospital, Sullia, Karnataka, India. ${ }^{2}$ Dr. Abhijit Kumar, Senior Resident, Department of Ophthalmology, Kasturba \\ Medical College, Manipal, Karnataka.
}

Corresponding Author: Dr. Abhijit Kumar, Senior Resident, Department of Ophthalmology, Kasturba Medical College, Manipal, Karnataka. E-mail id: draiyappads@gmail.com

\begin{abstract}
Aim: To study various corneal complications in manual small incision cataract surgery (MSICS), to study the risk factors leading to such complication and to study the final visual outcome following complications. Design:It is a prospective hospital based observational study. Methodology: In our study 100 eyes of 100 patients were studied who underwent MSICS with posterior chamber intraocular lens implantation. Corneal complications, their risk factors and final visual outcome were studied on post operatively day 1, day 8 and 6th week after surgery. Results: One hundred eyes of 100 patients who underwent MSICS were studied. Corneal complications were seen in $18 \%$ of patients. Of these $8 \%$ developed striate keratopathy, $6 \%$ developed corneal edema with $<10$ Descemet's folds, 3\% developed corneal edema with $>10$ Descemet's folds, $1 \%$ developed Descemet's membrane detachment. $94 \%$ of the patients categorized under good visual outcome category (BCVA better than 6/12), 89\% had best corrected visual acuity (BCVA) of 6/9 or better at the end of $6^{\text {th }}$ postoperative week. Conclusion: MCIS is associated with few corneal complications which cannot be overlooked. With appropriate management, most of the corneal complications will be resolved by $2^{\text {nd }}$ postoperative week. Vigilant attitude by the surgeon and timely management can reduce such complications and help in early visual rehabilitation.
\end{abstract}

Key words: Corneal complication, Cataract, Manual small incision cataract surgery, Striate keratopathy.

\section{Introduction}

In India, there are 12.5 million blind and it is estimated that $50 \%$ to $80 \%$ of them are blind due to cataract. In addition to the backlog, an additional 3.8 million become blind each year due to cataract [1]. The prevalence of visually significant cataracts and the rate of cataract surgery have rapidly increased. The Collaborative eye disease prevalence study predicts a $50 \%$ increase in the rate of cataract surgery over next two decades from 6.7 million patients in 2000 to 10 million patients in 2020 .

Therefore, a low rate of complications can still affect large number of patients [2]. With advances in technology and refinement in surgical techniques, the rate of complications secondary to cataract surgery has decreased. However, this has led to various new corneal complications like epithelial toxicity, mechanical or

Manuscript received: $4^{\text {th }}$ May 2019

Reviewed: $14^{\text {th }}$ May 2019

Author Corrected: $19^{\text {th }}$ May 2019

Accepted for Publication: $23^{\text {rd }}$ May 2019 toxic injury to endothelium, stripped Descemet's membrane, sterile corneal ulcer etc. Vigilance during cataract surgery and in postoperative period can prevent most complications. Surgical techniques for cataract extraction have undergone major evolution-from intracapsular extraction to phacoemulsification. Phacoemulsification offers desirable anatomical and functional results following cataract surgery and has become gold standard procedure in developed countries $[3,4]$.

In developing countries such as India, where there is a cataract backlog, Manual Small Incision Cataract Surgery (MSICS) with intraocular lens (IOL) implantation promises to be a cost-effective alternative to phacoemulsification [5]. Aim of cataract surgery is not only to restore visual acuity, but is now considered to be a refractive surgery. One of the major determinant of refractive status of the eye is cornea [6]. Preservation of corneal endothelial function continues to be a major 


\section{Original Research Article}

goal of cataract surgery. Postoperative visual outcome depends on the corneal complication sustain during the surgery [7]. Hence this study has been undertaken to highlight various corneal complications in manual small incision cataract surgery, to know the etiological factors leading to such complications and the final visual outcome.

\section{Materials and methods}

Following due permission from the Hospital Ethics Committee and written informed consent from patients, this prospective observational study comprising of 100 patients posted for Manual Small Incision Cataract Surgery (MSICS) with Intra Ocular Lens (IOL) implantation was conducted in rural hospital attached to K.V.G. Medical College, Sullia, Karnataka. All the Surgeries were conducted by single surgeon between November 2016 -August 2017

Inclusion criteria: Patients between 30 to 70 years of age, of either sex who were clinically diagnosed with senile cataract (mature/immature).

Exclusion criteria: Patients with traumatic cataract, congenital cataract, pseudo exfoliation syndrome, evidence of old iridocyclitis, glaucoma or corneal scars, previous intraocular surgery, diabetes mellitus, hypertension, high degree of refractive error.

Sample size: Previous studies [10] showed the prevalence of corneal complications after cataract surgery to be $5-7 \%$. Taking $7 \%$ as prevalence with Absolute precision of 0.05 at $95 \%$ confidence level, sample size worked out to be 80 , we recruited 100 patients.

Statistical analysis: Performed using the statistical package SPSSv19.0 [IBM India Pvt Ltd, Bangalore, India]. The categorical data were represented as numbers and percentages. The data collected were analysed for normal distribution by one-way analysis (and were normally distributed). Chi-square test was used for categorical data. Significance was defined as $\mathrm{p}$ value $<0.05$.

On the day of admission thorough preoperative evaluation was done which consisted of

1. Visual acuity.

2. Slit lamp bio microscopy with emphasis on type of cataract, gradingof nucleus, pupil size was done.

3. Direct and Indirect ophthalmoscopy, for posterior segment evaluation.

4. Applanation tonometry.
5. B-Scan for mature cataracts.

6. Keratometry reading were taken using Bausch and Lomb keratometer and biometry using Biomedix Echorule II for calculation of IOL power.

7. Systemic evaluation and physician fitness for surgery.

Informed consent was taken from all the patients prior to surgery. On the previous day of surgery, patients were advised to in still antibiotic drops and xylocaine test dose was given.

On the day of surgery, both upper and lower lid lashes were trimmed and the eye to be operated upon was dilated using tropicamide $(0.8 \%)$ and phenylephrine (5\%) eye drops. 1 drop every 10-15 minutes was instilled, starting one hour prior to surgery till full dilatation. One drop of Flurbiprofen $(0.03 \%)$ was instilled to prevent miosis and as a prophylaxis for prevention of cystoid macular edema. Under all aseptic precautions, MSICS with PCIOL fixation was done under peribulbar anaesthesia. 2\% Lignocaine with adrenaline 1:1000 along with Hyaluronidase was used. Hyaluronidase helps in spreading local anaesthetic in tissue spaces. About $5 \mathrm{ml}$ of local anaesthetic was injected. Digital massaging was given to spread local anaesthetic and achieve mild ocular hypotony.

After taking all aseptic precautions eye lids were draped, wire speculum placed, superior rectus bridle suture was passed and clamped on to the towel. Surgery was done under operating microscope Carl Zeiss S7

Procedure for manual small incision cataract surgery- A fornix based conjunctival flap of about 7 to $8 \mathrm{~mm}$ was made. Haemostasis was achieved using bipolar cautery. 6 to $6.5 \mathrm{~mm}$ scleral incision was made with number 15 blade of about one third of sclera thickness. Sclero corneal tunnelling done using crescent blade. Side port incision was taken at 9 O'clock position using $15^{\circ}$ side port entry blade in clear cornea about 1 $\mathrm{mm}$ from the limbus.

Air was injected into anterior chamber through side port. Anterior capsule (AC) was stained with trypan blue $(0.06 \%)$ after diluting it under the air bubble. Air was then replaced with $2 \%$ Hydroxy propyl methyl cellulose. Capsulorrhexis of about $6 \mathrm{~mm}$ in diameter was performed using bent $26 \mathrm{G}$ needle followed by gentle hydro dissection.

Nucleus was prolapsed into AC using Sins key hook and delivered by visco expression. Cortical clean-up was done using Simcoe's cannula. Finally, single piece 


\section{Original Research Article}

Poly Methyl Methacrylate IOL of $6 \mathrm{~mm}$ optic size was placed in capsular bag. During each of the intraocular steps adequate viscoelastic substance (2\% HPMC) was used to protect the corneal endothelium and maintain the anterior chamber.

At the end of the procedure, anterior chamber was formed with balanced salt solution, sub conjunctival injection of gentamicin with dexamethasone was given and eye covered with sterile pad. Operative time for MSICS were recorded. Any intra operative complication occurring during surgery was recorded.
Visual acuity and Slit lamp examination was done on first postoperative day in all patient and corneal complication if any were recorded. All patients received topical antibiotic steroid drops, which was used hourly for 1 week then reduced to 8 times a day for next 3 weeks then tapered over 2 weeks. The patients were reviewed on $8^{\text {th }}$ post op day and sixth postop week. During each visit uncorrected and best corrected visual acuity were recorded and a slit lamp examination was done to note the corneal status, intraocular lens position, postoperative inflammation and status of posterior segment.

\section{Results}

This study included 100 eyes of 100 patients with cataract who were admitted for MSICS with PCIOL implantation in rural hospital attached to K V G Medical College and Hospital, during the study period. A high follow up rate was achieved with a strong post-operative counselling process in the hospital.

The observations are as following

The majority of the patients who underwent MSICS were in age group of 50-59 years (36\%) and 60-69years (44\%) which constituted around $80 \%$ of total study group. The sex ratio and laterality of operated eye were comparable [Table 1]

Table-1: Patient characteristics, laterality, visual acuity and type of cataract.

\begin{tabular}{|c|c|c|}
\hline & Number of cases & Percentage \\
\hline \multicolumn{3}{|l|}{ 1. Age group (years) } \\
\hline $30-39$ & 03 & $3 \%$ \\
\hline $40-49$ & 17 & $17 \%$ \\
\hline $50-59$ & 36 & $36 \%$ \\
\hline $60-69$ & 44 & $44 \%$ \\
\hline \multicolumn{3}{|l|}{ 2. Sex distribution } \\
\hline Male & 47 & $47 \%$ \\
\hline Female & 53 & $53 \%$ \\
\hline \multicolumn{3}{|l|}{ 3. Laterality of the operated eye } \\
\hline Right Eye & 54 & $54 \%$ \\
\hline Left Eye & 46 & $46 \%$ \\
\hline \multicolumn{3}{|c|}{ 4. Preoperative uncorrected visual acuity } \\
\hline$<6 / 60$ & 20 & $20 \%$ \\
\hline $\mathrm{CF}^{*} 5^{1 / 2} / 2$ meters $-\mathrm{CF} 3 \frac{1}{1} 2$ meters & 34 & $34 \%$ \\
\hline CF 3 meters - CF $1 / 2$ meters & 30 & $30 \%$ \\
\hline
\end{tabular}


Original Research Article

\begin{tabular}{|l|c|c|}
\hline $\mathrm{HM}^{\dagger}$ close to face $-\mathrm{PL}^{\ddagger}$ positive & 16 & $16 \%$ \\
\hline 5. Type of cataract & 36 & $36 \%$ \\
\hline $\mathrm{NS}^{\S}$ grade $1 \& 2$ & 24 & $24 \%$ \\
\hline NS grade 3\&4 & 22 & $22 \%$ \\
\hline Mature cataract & 18 & $18 \%$ \\
\hline Cortical cataract & & \\
\hline
\end{tabular}

*Counting Fingers, ${ }^{\dagger}$ Hand Movements, ${ }^{\star}$ Perception of Light, ${ }^{\S}$ Nuclear Sclerosis

Out of 100 MSICS, 6 patients had intraoperative constriction of pupil due to iris touch with instruments, irrigation aspiration cannula, etc., 4 patients had posterior capsular rent, 2 patients had persistent iris prolapse and 1 patient had Descemet's membrane detachment. Remaining 86patients had no intra operative complication during surgery [Table 2].

Table-2: Intraoperative complications.

\begin{tabular}{|c|c|c|}
\hline Intra operative complication & Number of cases & Percentage \\
\hline Constriction of pupil & 6 & $6 \%$ \\
\hline Posterior capsular rupture & 4 & $4 \%$ \\
\hline Persistent iris prolapse & 3 & $3 \%$ \\
\hline Descemet's detachment & 1 & $1 \%$ \\
\hline Nil & 86 & $86 \%$ \\
\hline
\end{tabular}

Most common corneal complication in our studywas striate keratopathy, which resolved with treatment [Graph 1]

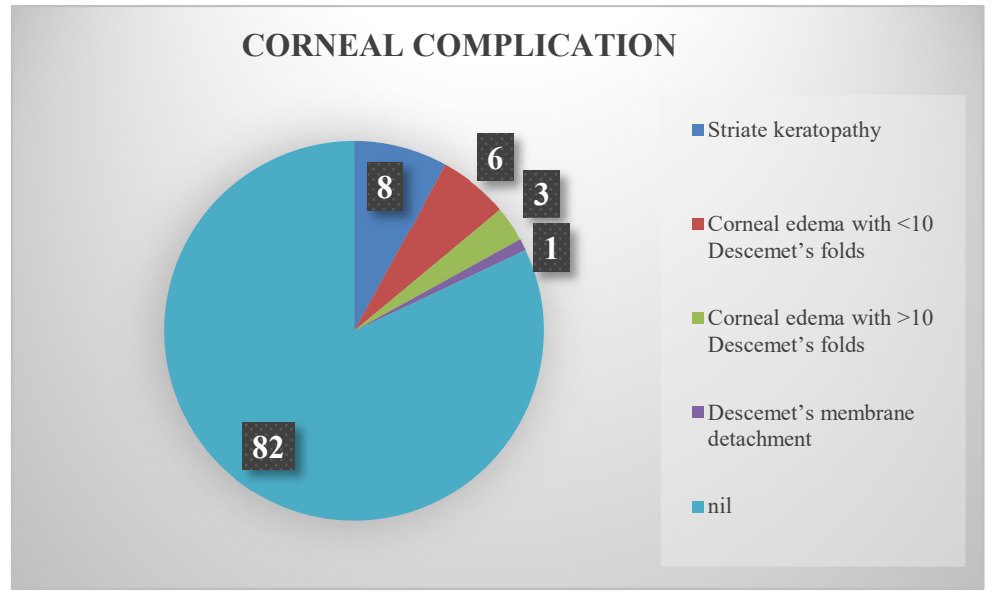

Graph 1: Corneal complications.

In the present study the following risk factors were assessed for corneal complications [Table 3]

Table-3: Risk factors for corneal complications

Risk factor

Increased maneuvering in $\mathrm{AC}$

\section{Complications}

P value

Present Absent

Present $18 \quad 04 \quad \mathrm{p}=<0.001$




\section{Original Research Article}

\section{Difficulty in prolapsing hard nucleus}

Difficulty in maneuvering in left eye surgery compared to right eye by right handed surgeon

Usage of Blunt instruments

Prolonged surgery

Excessive irrigation

$\begin{array}{llll}\text { Absent } & 00 & 78 & \\ \text { Present } & 10 & 04 & \mathrm{p}=<0.001 \\ \text { Absent } & 08 & 78 & \\ \text { Present } & 08 & 08 & \mathrm{p}=<0.001 \\ \text { Absent } & 10 & 74 & \\ \text { Present } & 06 & 00 & \mathrm{p}=<0.001 \\ \text { Absent } & 12 & 82 & \\ \text { Present } & 04 & 00 & \mathrm{p}=<0.001 \\ \text { Absent } & 14 & 82 & \\ \text { Present } & 07 & 02 & \mathrm{p}=<0.001 \\ \text { Absent } & 11 & 80 & \end{array}$

Out of 100 patients studied 18 patients with corneal complications had increased manoeuvring in anterior chamber and in 10 patients, we encountered difficulty in prolapsing hard nucleus and these two were the leading cause of corneal complication during MSICS.

Table-4: Post-operative uncorrected visual acuity $\left(8^{\text {th }}\right.$ post op day).

\begin{tabular}{|c|c|c|c|}
\hline \multirow{2}{*}{} & \multirow{2}{*}{ Post op day 1 } & \multicolumn{2}{|c|}{ Number of cases } \\
\cline { 2 - 4 } & 12 & Post op day 8 & End of 6 $^{\text {th }}$ week $^{*}$ \\
\hline $6 / 6-6 / 9$ & 44 & 48 & 68 \\
\hline $6 / 12-6 / 18$ & 38 & 22 & 22 \\
\hline $6 / 24-6 / 36$ & 6 & 2 & 10 \\
\hline$\leq 6 / 60$ & 6 & 0 \\
\hline
\end{tabular}

On the $1^{\text {st }}$ post-operative day, majority of the patients $(44 \%)$ had UCVA of $6 / 12$ to $6 / 18$, by the end of $6^{\text {th }}$ week majority (68\%) had UCVA of 6/6-6/9. [Table 4]

Table-5: Best Corrected Visual Acuity (BCVA) at the end of $6^{\text {th }}$ week.

\begin{tabular}{|c|c|c|}
\hline BCVA at the end of $\mathbf{6}^{\text {th }}$ week & Number of cases & Percentage \\
\hline $6 / 6$ & 68 & 21 \\
\hline $6 / 9$ & 21 & 5 \\
\hline $6 / 12$ & 5 & 4 \\
\hline $6 / 18$ & 4 & 2 \\
\hline $6 / 24$ & 2 & 0 \\
\hline $6 / 36$ & 0 & 0 \\
\hline $6 / 60$ & 0 & 0 \\
\hline
\end{tabular}

At the end of $6^{\text {th }}$ postoperative week, majority (68\%) of the patient attained best corrected visual acuity of $6 / 6$ and none had a vision worse than $6 / 36$ [Table 5].

\section{Discussion}

Corneal complications following cataract surgery and intraocular lens implantation continue to be more unusual because of advances in surgical techniques. The newer techniques have also led to various new corneal complications. Endothelial cell survival after cataract extraction and lens implantation are still major concerns. Hwang et al analysed corneal complications of cataract surgery and reported that the intraoperative and postoperative complications of cataract surgery that affect the cornea vary in aetiology and severity. Further, corneal edema as a result of cataract and/or intraocular lens surgery, has become a major cause of poor visual outcomes and a leading indication for keratoplasty Principal complications includes epithelial disruption, infections, sterile corneal ulceration, stromal melt, mechanical or toxic 
injury of the endothelium, vitreous touch, Descemet's membrane stripping, and epithelial and fibrous down growth [7] Meticulous cataract surgery with careful attention to protect the cornea can prevent most serious corneal complications.

In India, where cataract backlog is still a socio- economic problem, phacoemulsification remains an expensive modality of cataract extraction and difficult to afford to majority of population (mainly rural). MSICS has become very popular technique of cataract surgery in India, as it is not only safe and economical but easy for the majority of ophthalmologists to master. It is often used as an alternative to phacoemulsification. The considerable handling inside the anterior chamber during nucleus delivery increase the chances of iris injury, striate keratopathy, and PCR. The surgeon has to be extra careful in the construction of the scleral tunnel and to achieve a good capsulorrhexis. Postoperative inflammation and corneal edema are rare, if surgeons have the expertise and patience.

Two randomized controlled trial conducted in India by Sudhakar J et al and Venkatesh R et al, have found MSICS to be more effective and economical than ECCE and almost as effective and more economical than phacoemulsification [9] [10]. It has similar advantages to phacoemulsification in the rehabilitation of the cataract blind. It is also easier for a surgeon to train and master MSICS than phacoemulsification. There is no dependence on expensive machine and has less steep learning curve than that of phacoemulsification. Thus MSICS is ideal among small incision surgeries, for developing countries.

Sudhakar et al, analyzed the complications in 1000 cases of small incision cataract surgery with PCIOL implantation and reported that the most common early post- operative complication was striate keratopath y (7.3\%) followed by corneal edema1 (7\%) and iritis (4.8\%) [9]. A study conducted by Venkatesh et al, included 100 patients who underwent small incision cataract surgery at Aravind eye hospital, Pondicherry between May 03- Dec 03. On the $1^{\text {st }}$ postoperative day, 6 eyes $(6 \%)$ developed corneal oedema with $>10$ Descemets folds and 7 eyes $(7 \%)$ had corneal oedema with $<10$ Descemets folds. Corneal edema resolved with medical therapy by the time of discharge ( $3^{\text {rd }}$ day).

The cause of which was found to be hard cataract [10]. Gogate P.M., Kulkarni, S.R.et al observed that postoperative corneal edema was present in $4.5 \%$ of cases on $1^{\text {st }}$ day in phaco patients and $2 \%$ of small incision cataract surgery patients [11]

One hundred cases of Manual Small Incision Cataract Surgery were studied in terms of intra and postoperative corneal complications and visual outcome. Striate keratopathy and corneal edema were the common corneal complications encountered. Striate keratopathy was transient and resolved by the end of $1^{\text {st }}$ postop week. Corneal edema with $>10$ Descemet's folds resolved gradually with use of topical medication and was clear by the end of $6^{\text {th }}$ post-operative week. Kanski mentioned that corneal edema following cataract surgery is usually transient and often caused by intraoperative trauma to the endothelium by contact with instruments, lens matter or the intraocular lens. Complicated and prolonged surgery along with postoperative intraocular pressure spike may also contribute to this [12].

Table-6: Comparison of corneal complications between various studies.

\begin{tabular}{|l|c|c|c|}
\hline Complications & Sudhakar J et al & Venkatesh R et al & Present study \\
\hline Striate keratopathy & $7.3 \%$ & - & $8 \%$ \\
\hline Corneal edema with $<10$ Descemet's folds & - & $7 \%$ & $6 \%$ \\
\hline Corneal edema with $>10$ Descemet's folds & - & $5 \%$ & $3 \%$ \\
\hline Descemet's detachment & $0.1 \%$ & - & $1 \%$ \\
\hline
\end{tabular}

Our findings were comparable with the study conducted by Sudhakar $\mathrm{J}$ et al and Venkatesh $\mathrm{R}$ et al whereas DM detachment was present in 1 patient $[9,10]$. It did not resolve completely although the final corrected visual acuity being fairly good [table 6].

The reason here being -

$>$ Use of blunt entry blades

$>$ Increased maneuvering in $\mathrm{AC}$ 
$>$ Difficulty in prolapsing hard nucleus

$>$ Prolonged surgery

$>$ Excessive irrigation

The complications could have been avoided by -

$>$ Decreased maneuvering in $\mathrm{AC}$

$>$ Maintaining a distance from corneal endothelium by use of high molecular weight viscoelastic

$>$ Use of new and sharp entry blades

$>$ complete wash of viscoelastic substance at the end of procedure

$>$ Complete wash of cortical matter

$>$ Lesser infusion volume and minimum irrigation

$>$ Preventing repeated shallowing of AC

One of the World Health Organization quality indicator for cataract surgery is that, at least $85 \%$ of the patient operated should achieve best corrected visual acuity (BCVA) of $6 / 18$ or better [13]. In our study $98 \%$ of the patient had best visual acuity of 6/18 and better [Table 7].

Table-7: Comparison of BCVA at $6^{\text {th }}$ week between various studies.

\begin{tabular}{|c|c|c|c|}
\hline${\text { BCVA end of } \mathbf{6}^{\text {th }} \text { week }}^{\text {Venkatesh R et al [10] }}$ & Sudhakar J et al [9] & Present study \\
\hline $6 / 6$ & $66 \%$ & $33.3 \%$ & $68 \%$ \\
\hline $6 / 9$ & $28 \%$ & $23.8 \%$ & $21 \%$ \\
\hline $6 / 12$ & $5 \%$ & $23.6 \%$ & $5 \%$ \\
\hline $6 / 18$ & 0 & $7.9 \%$ & $2 \%$ \\
\hline $6 / 24$ & $1 \%$ & $3.8 \%$ & 0 \\
\hline $6 / 36$ & 0 & $1.4 \%$ & 0 \\
\hline $6 / 60$ & 0 & $1.0 \%$ & 0 \\
\hline$<6 / 60$ & 0 & $2.2 \%$ & 0 \\
\hline
\end{tabular}

Our study was comparable with Venkatesh $\mathrm{R}$ et al with respect to BCVA at the end of $6^{\text {th }}$ week. The study conducted by Sudhakar J et al had less patient with BCVA of $6 / 6$ at the end of $6^{\text {th }}$ week, this may be due the inclusion of patient with various associated conditions like myopia, diabetes mellitus, complicated cataracts, traumatic cataracts and developmental cataracts [9].

MSICS technique is good in terms of visual acuity due to less complication and better self-sealing sutureless incision compared to older techniques

Summary- In this study 100 cases of Manual Small Incision Cataract Surgery with PCIOL implantation were studied in terms of intra and postoperative corneal complications, risk factors involved and their final visual outcome. In developing countries like India where phacoemulsification is unaffordable to the majority of population requiring cataract surgery, MSICS could be safe and efficacious alternative technique of cataract surgery in rural hospital setting.

Although MSICS is associated with complications, meticulous surgery with careful attention in protecting the cornea can prevent it.

Recommendations- MSICS is a safe, affordable, efficacious alternative technique of cataract surgery in rural hospitals. It may be associated with fewerpostsurgical corneal complications, but appropriate and timely management of these complications achieves good post-operativevisual outcomes. 
Current study adding to existing knowledge- Manual small incision cataract surgery is a good and safe technique when performed keeping corneal endothelium away from the work plane and is cost effective and saves time when large number of cases has to be operated.

Authors contributions- KripaliniS H: Conceived, Designed, Data collection, manuscript writing and final approval of manuscript. Abhijit Kumar: Statistical analysis, Literature search and Manuscript editing.

Funding: Nil, Conflict of interest: Nil

Permission from IRB: Yes

\section{References}

1. RajshekharVemparala, Promila Gupta. National Programme for Control of Blindness (NPCB) in the 12th Five-year plan:An Overview.DJO2017;27:290-292

2. Park K. Parks text book of preventive and social medicine: Health Programmes in India. 22nd edition. Jabalpur:m/sBanarsidas BhanotPublishers;2013.p.405-8

3. Kershner RM. Sutureless one-handed intercapsular phacoemulsification. The keyhole technique. J Cataract Refract Surg. 1991;17 Suppl:719-25.

4. Gogate P. Comparison of various techniques for cataract surgery, their efficacy, safety, and cost. Oman J Ophthalmol 2010;3:105-6.

5. Gogate PM, Kulkarni SR, Krishnaiah S, wt al. Safety and efficacy of phacoemulsification compared with manual small-incision cataract surgery by a randomized controlled clinical trial: six-week results. Ophthalmology.2005 May;112(5):869-74.DOI:10.1016 / j.ophtha. 2004.11.055

6. Holladay JT, Cravy TV, Koch DD. Calculating the surgically induced refractive change following ocular surgery.J Cataract Refract Surg. 1992 Sep;18(5):429-43.

7. Hwang DG, Smith RE. Corneal complications of cataract surgery. Refract Corneal Surg. 1991 Jan-Feb; 7 (1): $77-80$.
8. Lumme P, Laatikainen LT. Risk factors for intraoperative and early postoperative complications in extracapsular cataract surgery. Eur J Ophthalmol. 1994 Jul-Sep; 4(3):151-8.

9. Sudhakar J, Ravindran RD, Natchiar G. Analysis of complications in 1000 cases of posterior chamber intra ocular lens implantation. Indian J Ophthalmol. 1989 Apr-Jun;37(2):78-9.

10. Venkatesh R, Das M, Prashanth S, et al. Manual small incision cataract surgery in eyes with white cataracts. Indian J Ophthalmol. 2005 Sep; 53 (3): 173-6.

11. Gogate PM. Small incision cataract surgery: Complications and mini-review. Indian $\mathrm{J}$ Ophthalmology 2009 Jan-Feb;57(1):45-9.

12. Kanski, Jack J. Clinical ophthalmology: a systematic approach: Management of age-related cataract. 6th edition. Edinburgh; Elsevier ButterworthHeinemann; 2007.p.360.

13. Baltussen R, Sylla M, Mariotti SP. Costeffectiveness analysis of cataract surgery: a global and regional analysis. Bull World Health Organ. 2004 May; 82 (5):338-45.

14. Baltussen R, Sylla M, Mariotti SP. Costeffectiveness analysis of cataract surgery: a global and regional analysis. Bull World Health Organ. 2004 May; $82(5): 338-45$.

How to cite this article?

Kriaplini S.H, Kumar A. A prospective study on corneal complications of small incision cataract surgery conducted in rural hospital. Trop J Ophthalmol Otolaryngol.2019;4(2): 92-99. doi: 10.17511/jooo.2019.i02.05 